\section{Comunidades quilombolas de Vitória da Conquista, Bahia, Brasil: autoavaliação de saúde e fatores associados}

\author{
Slave-descendent communities in Vitória da \\ Conquista, Bahia State, Brazil: self-rated \\ health and associated factors
}

\section{Comunidades quilombolas en Vitória da Conquista, Bahía, Brasil: autopercepción de salud y factores asociados}

\author{
1 Universidade Federal da \\ Bahia, Vitória da Conquista, \\ Brasil. \\ 2 Universidade Federal de \\ Minas Gerais, Belo Horizonte, \\ Brasil. \\ 3 Faculdade da Saúde \\ e Ecologia Humana, \\ Vespasiano, Brasil. \\ Correspondência \\ C. N. Kochergin \\ Universidade Federal da \\ Bahia. \\ Rua Rio de Contas 58 , \\ Quadra 17, Lote 58, Vitória \\ da Conquista, $B A$ \\ 45029-094, Brasil. \\ clavdian@yahoo.com.br
}

\begin{abstract}
This study aimed to analyze the prevalence of negative self-rated health and associated factors in the quilombola community (descendants of escaped slaves) in Vitória da Conquista, Bahia State, Brazil. A household survey was conducted with 797 adults in 2011. Data on self-rated health, socioeconomic and demographic characteristics, lifestyle, social support, health status, and access to health services were obtained through questionnaires. Multivariate logistic regression analysis was performed, adjusted for sex and age. Prevalence of negative self-rated health was $12.5 \%$. After statistical modeling, the following variables remained associated with self-rated health: skin color, schooling, adequate consumption of fruits and vegetables, chronic illness, physical limitations, and at least one medical visit in the previous 12 months. Selfrated health was associated with socioeconomicl demographic dimensions, lifestyle, social support, and health status.
\end{abstract}

African Continental Ancestry Group; SelfAssessment; Health Status
Clavdia Nicolaevna Kochergin 1,2

Fernando Augusto Proietti 2,3

Cibele Comini César 2

\section{Resumo}

Este estudo objetiva analisar a prevalência da autoavaliação de saúde negativa e fatores associados na população quilombola de Vitória da Conquista, Bahia, Brasil. Realizou-se um inquérito domiciliar com 797 adultos, em 2011. Os dados sobre autoavaliação de saúde, condições socioeconômicas e demográficas, estilo de vida, suporte social, situação de saúde e acesso a serviço de saúde, foram obtidos por meio da aplicação de questionários. A análise multivariável foi realizada por regressão logística ordinal, ajustada por sexo e idade. A prevalência de autoavaliação negativa de saúde foi de 12,5\%. Após modelagem estatística as seguintes variáveis permaneceram associadas à autoavaliação de saúde: cor, escolaridade, consumo satisfatório de frutas e verduras, presença de doença crônica, limitações físicas, apoio social e ao menos uma consulta médica nos últimos 12 meses. A autoavaliação de saúde foi associada às dimensões: socioeconômica/demográfica, hábitos de vida, suporte social e situação de saúde.

Grupo com Ancestrais do Continente Africano; Autoavaliação; Nível de Saúde 


\section{Introdução}

A autoavaliação da saúde é um indicador complexo do estado de saúde do indivíduo que possui como atributo a capacidade de uma avaliação global, uma vez que traz consigo a apreensão tanto de aspectos objetivos quanto subjetivos da saúde 1,2. Passou a ser incorporado às pesquisas sobre condições de saúde populacionais há pouco mais de três décadas, respaldado por estudos que demonstraram sua robusta associação com a mortalidade, morbidade, limitação funcional, qualidade de vida e uso dos serviços de saúde $2,3,4,5,6$. É um conceito subjetivo que embora esteja fortemente relacionado a medidas biológicas objetivas é mediado por experiências psicossociais e pelos contextos sociais e culturais. Pode ser entendido como resultante de processo cognitivo, frequentemente intuitivo, que envolve processamento das informações, interpretação de significados e seleção.

Jylhä 3 propôs um modelo teórico para o processo de autoavaliação que envolve três estágios. No primeiro, a pessoa reconhece o significado de saúde e identifica quais os componentes relevantes para avaliar o seu estado de saúde; no segundo, avalia a importância de cada um destes componentes; por fim, decide qual dos níveis da escala predefinida melhor resume a sua situação.

No Brasil, os Suplementos de Saúde, incluídos nos anos 1998, 2003 e 2008 na Pesquisa Nacional por Amostra de Domicílios (PNAD), são importantes fontes de informação sobre a autoavaliação do estado de saúde ${ }^{5}$. Resultados da PNAD 2008 informam a prevalência global de 3,8\% para a autoavaliação de estado de saúde "ruim ou muito ruim", variando de $4,6 \%$ na Região Nordeste a $3,3 \%$ na Sudeste 5 . Verifica-se variabilidade por situação de residência, com maior percentual de avaliação negativa na zona rural tanto no país como um todo quanto no Estado da Bahia (Ministério da Saúde. PROADESS: Avaliação do Desempenho do Sistema de Saúde. http://www.proadess.icict.fiocruz.br, acessado em 25/Jul/2012).

Estudos de base populacional conduzidos nos últimos anos no país verificaram associação entre a autoavaliação da saúde e fatores sociodemográficos, medidas objetivas de saúde, fatores comportamentais e psicossociais. Foi observado que a saúde tende a ser pior avaliada entre mulheres, idosos, pessoas com piores condições de saúde, com menor acesso ao serviço de saúde, de menor escolaridade, menor renda, com hábitos de vida não saudáveis e com pouco suporte social 7,8,9,10. Internacionalmente, fatores como pertencimento a minorias étnicas e raciais, falta de apoio social, ser imigrante, entre outros, têm sido relacionados à pior autoavaliação de saúde 11,12,13,14. Além disso, constatou-se diferença entre os sexos com relação à importância atribuída aos fatores, sendo os psicossociais mais importantes para as mulheres e os comportamentais para os homens 15 .

No Brasil, país com o maior contingente de população negra fora da África 16, é recente o esforço no sentido de compreender os determinantes sociais de saúde priorizando a população negra. Diferentes estudos expuseram a situação de marginalização socioeconômica e a precariedade das condições de vida e de saúde desse contingente populacional: sobrerrepresentação nos índices de pobreza, menor expectativa de vida ao nascer, menor acesso aos serviços de saúde, maior mortalidade infantil e materna, pior percepção do estado de saúde em comparação com a população branca 16,17,18,19,20. Diferentemente de outros países, por exemplo, os Estados Unidos, são ainda raras as publicações nacionais que buscam a interconexão étnicoracial e iniquidades em saúde 21,22. Entre as dificuldades para a incorporação do recorte étnicoracial na área da saúde, estão as questões de ordem teórico-metodológicas envolvendo definições e operacionalização dos conceitos etnia, raça e de suas classificações 21,22,23,24. No tocante às desigualdades étnico-raciais, a situação dos quilombolas merece destaque por se tratar de um grupo em situação de vulnerabilidade social, decorrente de um processo histórico de resistência à escravidão e invisibilidade política $\mathrm{e}$ social, do pós-abolicionismo ao reconhecimento na Constituição Federal de 1988, cujo reflexo está expresso em piores indicadores sociais e de saúde 19,25 .

Entende-se por remanescentes de quilombos o que está no Decreto Federal no 4.887/2003 26: "Consideram-se remanescentes das comunidades dos quilombos, os grupos étnico-raciais, segundo critérios de autoatribuição, com trajetória histórica própria, dotados de relações territoriais específicas, com presunção de ancestralidade negra relacionada com a resistência à opressão histórica sofrida".

Pensar a situação de saúde da população quilombola é, também, considerar a localização das comunidades que, situadas geralmente em áreas rurais, apresentam um relativo isolamento geográfico, aumentando o grau de exposição desta população às iniquidades em saúde e dificultando o acesso aos serviços de saúde 17,19,27.

Apesar de fazermos referência à "população quilombola", é importante ressaltar a necessidade de investigações conduzidas no sentido de buscar as especificidades dos diferentes quilombos existentes no país, pois estes guardam entre 
si diferenças não só geográficas como também relacionadas às trajetórias históricas próprias 22.

Políticas públicas têm sido propostas no país com o intuito de articular ações intersetoriais visando a reduzir a vulnerabilidade social em comunidades remanescentes de quilombos 27 . No entanto, a efetividade dessas ações carece de estudos demográficos e epidemiológicos que as subsidiem 18 .

Estudos envolvendo populações quilombolas foram conduzidos em diferentes estados do país e identificaram precárias condições socioeconômicas e presença de diversos agravos à saúde 17,18,19,27. Não foram encontrados trabalhos que caracterizem a situação de saúde da população de remanescentes de quilombos do Município de Vitória da Conquista, Bahia. Considerando que por meio da autoavaliação do estado de saúde é possível identificar necessidades de saúde de uma população 5,10,11, acredita-se que este estudo possa contribuir para a construção de um corpo de conhecimento sobre as condições de saúde dessa população em particular.

Este trabalho teve como objetivo estimar a prevalência de autoavaliação ruim/muito ruim nessa população e verificar os fatores demográficos, sociais, comportamentais e psicossociais associados à autoavaliação de saúde, estratificada por sexo.

\section{Metodologia}

\section{Desenho do estudo e coleta de dados}

Estudo transversal realizado em comunidades quilombolas certificadas pela Fundação Cultural Palmares 27, localizadas no Município de Vitória da Conquista, no período de setembro a outubro de 2011. Parte integrante do projeto de pesquisa COMQUISTA: Comunidades Quilombolas de Vitória da Conquista - BA. Avaliação de Saúde e seus Condicionantes, realizado pela Universidade Federal da Bahia (UFBA) e Universidade Federal de Minas Gerais (UFMG).

O Município de Vitória da Conquista está localizado na região econômica do Sudoeste da Bahia, possui a 5ạ maior economia do estado, com participação de $2,29 \%$ no Produto Interno Bruto (PIB) estadual. A população total é de 315.884 habitantes, sendo 10\% rural. Quanto à cor, 9,8\% dos habitantes são classificados como pretos e 55,2\% como pardos (Prefeitura Municipal de Vitória da Conquista. Descrição da População de Vitória da Conquista. http:// www.pmvcba.gov.br/v2/dados-estatisticos, acessado em 05/Abr/2013). Possui 25 comunidades remanescentes de quilombos certifica- das pela Fundação Cultural Palmares, localizadas na área rural do município e concentradas em cinco dos 11 distritos da região, distantes, em média, 31,2Km da sede do município. Suas origens remontam à permanência de escravos negros na região após a falência de empreendimentos agrícolas 25. Participaram da pesquisa cinco comunidades que atenderam aos critérios de inclusão: certificação pela Fundação Cultural Palmares e contar com pelo menos cinquenta famílias cadastradas.

A população de referência do trabalho consistiu de indivíduos com idade igual ou superior a 18 anos, residentes nas comunidades quilombolas incluídas no estudo, sendo estimada em 2.935 indivíduos. A amostragem foi feita em dois estágios: foi selecionada uma comunidade em cada distrito, e nas comunidades selecionadas foram escolhidos os domicílios. Todos os indivíduos com 18 anos ou mais residentes nos domicílios escolhidos foram convidados a participar da pesquisa.

O cálculo do tamanho amostral baseou-se nos seguintes parâmetros: prevalência de 50\%, precisão de $5 \%$, intervalo de $95 \%$ de confiança (IC95\%) e efeito de desenho igual a 2, perfazendo um total de 680 indivíduos. A esse valor acrescentou-se $30 \%$ para compensar as perdas, totalizando uma amostra de 884 indivíduos.

Neste estudo, foram excluídas as mulheres em período gestacional, por ser este período dotado de especificidades que podem alterar a autoavaliação do estado de saúde e sua associação com as variáveis explicativas, e os indivíduos para os quais as informações foram relatadas por informantes secundários, considerando que as informações autorrelatadas refletem melhor a condição de saúde do indivíduo do que as relatadas por informantes 28 .

Os dados foram coletados por meio de entrevistas individuais, realizadas por entrevistadores treinados pela equipe coordenadora do projeto. $\mathrm{O}$ instrumento de entrevistas utilizado foi uma versão adaptada para este trabalho do questionário semiestruturado da Pesquisa Nacional de Saúde (PNS). Detalhes sobre o desenho do estudo e coleta dos dados podem ser obtidos em Bezerra et al. 29 .

\section{Variáveis estudadas}

A autoavaliação da saúde, variável de desfecho, foi obtida por meio da pergunta: "Em geral, como o(a) sr(a) avalia a sua saúde?”, com as respostas em escala de cinco categorias (muito boa, boa, regular, ruim e muito ruim). Para a análise, as respostas foram categorizadas em três níveis: muito boa/boa, regular e ruim/muito ruim. 
As variáveis explicativas incluídas neste estudo foram: (1) sociodemográficas - condição de quilombola autorreferida (sim, não); cor autorreferida (preta, parda e outra, incluindo brancos, amarelos e indígenas); sexo (masculino, feminino); idade em grupo etário (18-29, 30-39, 40-49, 40-59, 60 e mais); escolaridade em anos completos de estudos agrupados (0, 1-3, 4-7, 8 e mais); situação conjugal [com companheiro(a), sem companheiro(a)]; possuir fonte de renda (sim, não); (2) hábitos relacionados à saúde - prática de atividade física (sim, não); tabagismo (nunca fumou, ex-fumante, fumante); consumo de álcool (nenhum, moderado, excessivo); consumo de frutas e verduras (satisfatório, insatisfatório); (3) situação de saúde - estado nutricional medido pelo índice de massa corporal (IMC) obtido com base no peso e altura aferidos e classificado em baixo peso (IMC $<18,5 \mathrm{~kg} / \mathrm{m}^{2}$ ), peso adequado $\left(18,5 \leq \mathrm{IMC}<25 \mathrm{~kg} / \mathrm{m}^{2}\right)$, sobrepeso $(25 \leq \mathrm{IMC}<$ $30 \mathrm{~kg} / \mathrm{m}^{2}$ ), obeso (IMC $\geq 30 \mathrm{~kg} / \mathrm{m}^{2}$ ); limitação nas atividades da vida diária (nenhuma, uma, duas, três ou mais); "sentir-se deprimido nos últimos 15 dias” (alguma vez, nunca), número de doenças crônicas (nenhuma, uma, duas, três ou mais), ter sofrido acidentes ou ferimentos nos últimos 12 meses (sim, não); (4) acesso a serviço de saúde - avaliado pela realização de consulta médica nos últimos 12 meses (sim, não); (5) apoio social (menor apoio, maior apoio); e (6) contexto domiciliar (piores condições, melhores condições).

Os indígenas foram incorporados na categoria "outra", juntamente com os brancos e amarelos, devido à sua pequena participação na amostra ( $n=20,2,68 \%$ ) e por apresentarem perfil de autoavaliação em saúde semelhante aos demais componentes do agrupamento, intermediário entre brancos e amarelos. O padrão de consumo de álcool foi construído utilizandose as informações de número de doses diárias e números de dias da semana em que havia consumo, gerando o consumo semanal. Esse foi classificado em consumo excessivo se maior que dez doses para mulher e 15 doses para homens, ou o consumo de cinco ou mais doses em uma única ocasião por homens ou quatro ou mais por mulheres, pelo menos uma vez nas últimas duas semanas. Para o consumo de sucos, frutas, legumes e verduras considerou-se o número de dias na semana em que consumiu pelo menos uma porção destes alimentos. Foi considerado satisfatório se $\geq 5$ dias. O número de doenças crônicas foi construído baseando-se nas informações autorreferidas do diagnóstico médico de 12 doenças crônicas (hipertensão arterial, diabetes, hipercolesterolemia, doença cardíaca, acidente vascular cerebral, asma ou bronquite, depressão, outra doença mental, doença pulmonar, artrite, problema na coluna e osteoporose. Não se considerou presença de hipertensão arterial ou diabetes exclusivamente durante gravidez). A variável acidentes e ferimentos foi construída com base nas respostas positivas para as questões: acidentes de trânsito, acidentes exceto de trânsito e violência física ou agressões que tenham resultado em lesões corporais nos últimos 12 meses.

O apoio social foi avaliado valendo-se das informações sobre familiares ou parentes com quem se sente à vontade e pode falar sobre quase tudo (um ou mais, nenhum); amigo(s) com quem se sente à vontade e pode falar sobre quase tudo (um ou mais, nenhum); frequência a grupos para atividades esportivas e/ou artísticas (muito frequente/frequente, raramente/nunca); frequência a reuniões de associação de moradores ou funcionários, sindicatos ou partidos, centros acadêmicos ou similares (muito frequente/frequente, raramente/nunca); frequência a cultos religiosos (muito frequente/frequente, raramente/nunca); realização de trabalhos voluntários (sim, não). Para cada uma das variáveis criou-se um indicador de participação/apoio. Com base nesses indicadores foi criado um escore de apoio social utilizando-se a técnica de análise fatorial, considerando como entrada a matriz de correlação tetracórica dos indicadores. O escore foi posteriormente categorizado pelo valor da mediana, em alto e baixo. O contexto domiciliar foi avaliado por meio do tipo de abastecimento de água (rede geral/poço/cisterna, tanque/açude/ aguada/outro); disponibilidade de rede elétrica (sim, não); destino do lixo (queimado/enterrado, jogado no rio/terreno/outro); material utilizado na construção da moradia (alvenaria/madeira aparada, taipa/barro/adobe/outros) e foi elaborado de maneira similar ao apoio social.

\section{Análise estatística}

Inicialmente, foi feita uma análise descritiva dos dados e estimada a prevalência em cada uma das categorias da autoavaliação do estado de saúde para a amostra total e estratificada por sexo, com os respectivos IC95\%. Procedeu-se a análise de associação da variável resposta com cada variável explicativa, ajustando por sexo e idade, utilizando-se o modelo logístico ordinal - modelo de chances proporcionais (MCP) 30 . Variáveis significativas no nível de $20 \%$ foram selecionadas para inclusão no modelo final. No modelo final, ajustado por idade e sexo, permaneceram aquelas que apresentaram significância no nível de $5 \%$. O modelo foi testado com relação à suposição de proporcionalidade das chances. Para as variáveis nas quais a suposição não foi verificada, a suposição foi relaxada com a adoção do mode- 
lo de chances proporcionais parciais (MCPP) 30 . A comparação entre modelos foi feita pelo teste da razão de verossimilhança e o ajuste do modelo final foi avaliado pelo teste de Hosmer \& Lemeshow.

Todas as análises foram realizadas com o auxílio do programa Stata, versão 12.0 (Stata Corp., College Station, Estados Unidos).

\section{Questões éticas}

O projeto foi aprovado pelos Comitês de Ética em Pesquisa da Faculdade São Francisco de Barreira (CAAE 0118.0.066.000-10) e da UFMG (CAAE 0118.0.066.203-10).

\section{Resultados}

Foram visitados 422 domicílios e identificados 943 indivíduos elegíveis para o estudo; 797 participaram da pesquisa. A perda de 146 indivíduos $(13,3 \%)$ deveu-se ao não encontro após três visitas ao domicílio ou recusa. Essas são referentes majoritariamente a indivíduos do sexo masculino ( $\mathrm{n}=113,77,4 \%$ ) e na faixa etária de 18 a 34 anos ( $n=95,65,2 \%)$. Para avaliar o efeito das perdas sobre as estimativas foi efetuada análise ponderada utilizando-se peso pós-estratificação e os resultados não diferiram da análise não ponderada. Optou-se por apresentar os resultados não ponderados.

Dos 797 indivíduos que concordaram em participar da pesquisa, foram excluídos 36 infor- mantes secundários $(4,5 \%), 11$ gestantes $(1,4 \%)$ e $3(0,4 \%)$ participantes que não responderam à questão relativa à autoavaliação de saúde, resultando em uma amostra de 747 indivíduos.

A distribuição da autoavaliação da saúde é apresentada na Figura 1, para a amostra total e segundo os sexos, com os respectivos IC95\%. Homens e mulheres não diferiram significativamente com relação à distribuição. Na amostra total, a prevalência de autoavaliação da saúde ruim/ muito ruim foi de 12,5\% (IC95\%: 10,1\%; 14,8\%). Homens apresentaram prevalência (ruim/muito ruim) de $14 \%$ (IC95\%: 10,4\%; 17,7\%) e mulheres de 11,1\% (IC95\%: 8,0\%; 14,1\%). A Tabela 1 apresenta a distribuição da autoavaliação da saúde segundo sexo sem agregação das categorias de resposta.

A Tabela 2 apresenta a distribuição das variáveis explicativas para a amostra total e para homens e mulheres. Os participantes autorreportaram-se majoritariamente como quilombolas $(83,5 \%)$, do sexo feminino $(53,3 \%)$, cor preta $(39,4 \%)$ ou parda $(44,6 \%)$, idades entre 18 e $29 \operatorname{anos}(24,4 \%)$, com companheiro $(63,9 \%)$, tinham de 1 a 3 anos de estudos (28\%) ou não tinham frequentado a escola $(32,4 \%)$ e possuíam alguma fonte de renda (71,7\%). A inatividade física foi relatada por $74,2 \%$ dos entrevistados, $19,8 \%$ informaram fumar e 58,6\% negaram o consumo de bebida alcoólica. Quanto ao consumo diário de frutas e verduras, 69,3\% informaram consumo satisfatório. Com relação às condições de saúde, identificou-se sobrepeso em 30,4\% dos indivíduos e obesidade em 10,7\%.

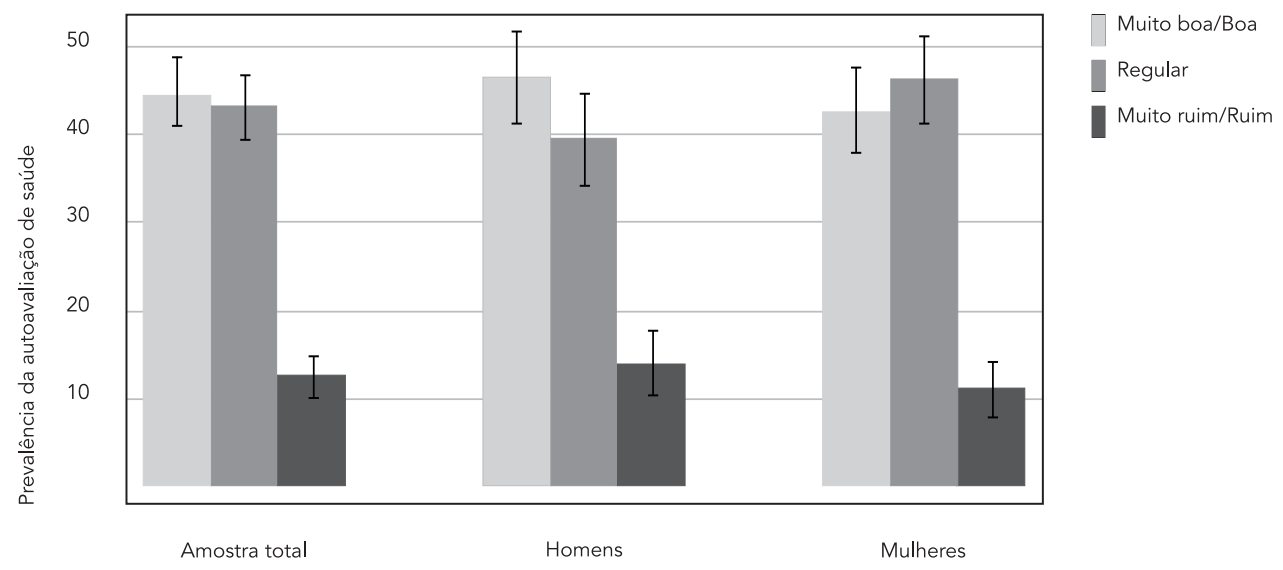


Tabela 1

Autoavaliação de saúde para amostra total e estratificada por sexo, segundo categoria de resposta. População quilombola, Vitória da Conquista, Bahia, Brasil, 2011.

\begin{tabular}{lcccccc}
\hline Autoavaliação de saúde & \multicolumn{2}{c}{ Total } & \multicolumn{2}{c}{ Homem } & \multicolumn{2}{c}{ Mulher } \\
& $\%$ & IC95\% & $\%$ & IC95\% & $\%$ & IC95\% \\
\hline Muito boa & 5,1 & 3,$5 ; 6,7$ & 6,0 & 3,$5 ; 8,5$ & 4,3 & 2,$3 ; 6,3$ \\
Boa & 39,4 & 3,$5 ; 6,7$ & 40,4 & 35,$2 ; 45,6$ & 38,4 & 33,$6 ; 43,2$ \\
Regular & 43,1 & 39,$5 ; 46,7$ & 39,5 & 34,$4 ; 44,7$ & 46,2 & 41,$3 ; 51,2$ \\
Ruim & 10,0 & 7,$9 ; 12,2$ & 11,7 & 8,$4 ; 15,1$ & 8,5 & 5,$8 ; 11,3$ \\
Muito ruim & 2,4 & 1,$3 ; 3,5$ & 2,3 & 0,$7 ; 3,9$ & 2,5 & 0,$9 ; 4,1$ \\
\hline
\end{tabular}

IC95\%: intervalo de $95 \%$ de confiança.

\section{Tabela 2}

Distribuição de variáveis demográficas, socioeconômicas, comportamentais e de saúde: amostra total, homens e mulheres. População quilombola, Vitória da Conquista, Bahia, Brasil, 2011.

\begin{tabular}{|c|c|c|c|c|}
\hline Variáveis & $\begin{array}{l}\text { Amostra total } \\
\begin{array}{c}(n=747) \\
n(\%)\end{array}\end{array}$ & $\begin{array}{c}\text { Homem } \\
\begin{array}{c}(n=349) \\
n(\%)\end{array}\end{array}$ & $\begin{array}{c}\text { Mulher } \\
\text { (n = 398) } \\
n(\%)\end{array}$ & Valor de $\mathrm{p}$ \\
\hline \multicolumn{5}{|l|}{ Quilombola } \\
\hline Sim & $623(83,5)$ & $283(81,3)$ & $340(85,4)$ & 0,132 \\
\hline Não & $123(16,5)$ & $65(18,7)$ & $58(14,6)$ & \\
\hline \multicolumn{5}{|l|}{ Idade (anos) } \\
\hline $18-29$ & $182(24,4)$ & $74(21,2)$ & $108(27,5)$ & 0,047 \\
\hline $30-39$ & $156(20,8)$ & $64(18,3)$ & $92(23,1)$ & \\
\hline $40-49$ & $147(19,7)$ & $72(20,6)$ & $75(18,9)$ & \\
\hline $50-59$ & $101(13,5)$ & $56(16,1)$ & $45(11,3)$ & \\
\hline 60 e mais & $161(21,6)$ & $83(23,8)$ & $78(19,6)$ & \\
\hline \multicolumn{5}{|l|}{ Sexo } \\
\hline Masculino & $349(46,7)$ & & & \\
\hline Feminino & $398(53,3)$ & & & \\
\hline \multicolumn{5}{|l|}{ Cor } \\
\hline Preta & $291(39,0)$ & $130(37,2)$ & $161(40,4)$ & 0,164 \\
\hline Parda & $329(44,0)$ & $150(44,0)$ & $179(45,0)$ & \\
\hline Outras & $127(17,0)$ & $69(19,8)$ & $58(14,6)$ & \\
\hline \multicolumn{5}{|l|}{ Escolaridade (anos) } \\
\hline 0 & $241(32,4)$ & $116(33,3)$ & $125(31,6)$ & 0,900 \\
\hline $1-3$ & $208(28,0)$ & $96(27,6)$ & $112(28,4)$ & \\
\hline $4-7$ & $206(27,7)$ & $93(26,7)$ & $113(28,6)$ & \\
\hline 8 e mais & $88(11,8)$ & $43(12,4)$ & $45(11,4)$ & \\
\hline \multicolumn{5}{|l|}{ Situação conjugal } \\
\hline Com companheiro(a) & $477(63,9)$ & $224(64,2)$ & $253(63,6)$ & 0,861 \\
\hline Sem companheiro(a) & $270(36,1)$ & $125(35,8)$ & $145(36,4)$ & \\
\hline \multicolumn{5}{|l|}{ Possuir fonte de renda } \\
\hline Sim & $526(71,7)$ & $233(68,1)$ & $293(74,7)$ & \\
\hline Não & $208(28,3)$ & $109(31,9)$ & $99(25,3)$ & 0,047 \\
\hline \multicolumn{5}{|l|}{ Prática de atividade física } \\
\hline Sim & $193(25,8)$ & $121(34,7)$ & $72(18,1)$ & $<0,001$ \\
\hline Não & $554(74,2)$ & $228(65,3)$ & $326(81,9)$ & \\
\hline
\end{tabular}

(continua) 
Tabela 2 (continuação)

\begin{tabular}{|c|c|c|c|c|}
\hline Variáveis & $\begin{array}{l}\text { Amostra total } \\
\begin{array}{c}(n=747) \\
n(\%)\end{array}\end{array}$ & $\begin{array}{c}\text { Homem } \\
\begin{array}{c}(n=349) \\
n(\%)\end{array}\end{array}$ & $\begin{array}{c}\text { Mulher } \\
\text { (n = 398) } \\
n(\%)\end{array}$ & Valor de $p$ \\
\hline \multicolumn{5}{|c|}{ Consumo de frutas/verduras } \\
\hline Satisfatório & $518(69,3)$ & $245(70,2)$ & $273(68,6)$ & 0,634 \\
\hline Insatisfatório & $229(30,7)$ & $105(29,8)$ & $125(31,4)$ & \\
\hline \multicolumn{5}{|l|}{ Estado nutricional } \\
\hline Peso adequado & $413(55,3)$ & $235(67,3)$ & $178(44,7)$ & $<0,001$ \\
\hline Baixo peso & $27(3,6)$ & $13(3,7)$ & $14(3,5)$ & \\
\hline Sobrepeso & $227(30,4)$ & $89(25,5)$ & $138(34,7)$ & \\
\hline Obeso & $80(10,7)$ & $12(3,5)$ & $68(17,1)$ & \\
\hline \multicolumn{5}{|l|}{ Consumo de álcool } \\
\hline Nenhum & $437(58,6)$ & $150(43,1)$ & $287(72,1)$ & $<0,001$ \\
\hline Moderado & $229(30,7)$ & $136(39,1)$ & $93(23,4)$ & \\
\hline Excessivo & $80(10,7)$ & $62(17,8)$ & $18(4,5)$ & \\
\hline \multicolumn{5}{|l|}{ Tabagismo } \\
\hline Nunca fumou & $395(52,9)$ & $111(31,8)$ & $284(71,4)$ & $<0,001$ \\
\hline Ex-fumante & $204(27,3)$ & $86(36,4)$ & $77(19,3)$ & \\
\hline Fumante & $148(19,8)$ & $63(31,8)$ & $37(9,3)$ & \\
\hline \multicolumn{5}{|c|}{$\begin{array}{l}\text { Número de doenças crônicas (com } \\
\text { diagnóstico médico) }\end{array}$} \\
\hline Nenhuma & $297(39,8)$ & $149(42,7)$ & $148(37,2)$ & 0,274 \\
\hline 1 & $253(33,9)$ & $112(32,1)$ & $141(35,4)$ & \\
\hline 2 & $125(16,7)$ & $60(17,2)$ & $65(16,3)$ & \\
\hline 3 ou mais & $72(9,6)$ & $28(8,0)$ & $44(11,1)$ & \\
\hline \multicolumn{5}{|c|}{ Limitação nas atividades da } \\
\hline \multicolumn{5}{|l|}{ vida diária } \\
\hline Nenhuma & $287(38,4)$ & $154(44,1)$ & $133(33,4)$ & 0,014 \\
\hline 1 & $137(18,3)$ & $64(18,3)$ & $73(18,4)$ & \\
\hline 2 & $108(14,5)$ & $45(12,9)$ & $63(15,8)$ & \\
\hline 3 ou mais & $215(28,8)$ & $86(24,7)$ & $129(32,4)$ & \\
\hline \multicolumn{5}{|c|}{ Sentir-se deprimido (últimos 15 dias) } \\
\hline Alguma vez & $101(13,5)$ & $43(12,3)$ & $58(14,6)$ & 0,369 \\
\hline Nunca & $646(86,5)$ & $306(87,7)$ & $340(85,4)$ & \\
\hline \multicolumn{5}{|c|}{ Acidentes/Ferimentos (últimos } \\
\hline \multicolumn{5}{|l|}{12 meses) } \\
\hline Sim & $80(10,7)$ & $44(12,6)$ & $36(9,1)$ & 0,116 \\
\hline Não & $667(89,3)$ & $305(87,4)$ & $362(90,9)$ & \\
\hline \multicolumn{5}{|c|}{ Consulta médica (últimos 12 meses) } \\
\hline Sim & $373(49,9)$ & $201(57,6)$ & $172(43,2)$ & $<0,001$ \\
\hline Não & $374(50,1)$ & $148(42,4)$ & $226(56,8)$ & \\
\hline \multicolumn{5}{|l|}{ Apoio social } \\
\hline Menor apoio & $241(67,7)$ & $137(39,3)$ & $104(26,1)$ & $<0,001$ \\
\hline Maior apoio & $506(32,3)$ & $212(60,7)$ & $294(77,9)$ & \\
\hline \multicolumn{5}{|l|}{ Contexto domiciliar } \\
\hline Piores condições & $375(50,2)$ & $165(47,3)$ & $210(52,8)$ & 0,135 \\
\hline Melhores condições & $372(49,8)$ & $184(52,7)$ & $188(47,2)$ & \\
\hline
\end{tabular}

Ausência de diagnóstico de doença crônica foi observada em $39,8 \%$ dos entrevistados, $38,4 \%$ não apresentavam limitação nas atividades da vida diária e 13,5\% relataram depressão nos 15 dias anteriores à pesquisa. Quanto à consulta médica nos últimos 12 meses, 50,1\% dos res- 
pondentes informaram ter realizado ao menos uma. Um maior apoio social foi identificado para $67,7 \%$ dos indivíduos e melhor contexto domiciliar para $49,8 \%$.

A distribuição das variáveis explicativas não foi igual para homens e mulheres. Os homens eram mais velhos, apresentaram maior prevalência de pessoas sem renda, de ativos no lazer, de fumantes e ex-fumantes, de consumidores de álcool, de adequação de peso, de ausência de limitação funcional, de vítimas de acidente e ferimentos nos últimos 12 meses, de uso do serviço de saúde e menor apoio social.

A Tabela 3 apresenta os resultados da análise de associação das variáveis explicativas com a autoavaliação em saúde, ajustada por idade e sexo. Para a amostra total, melhor autoavaliação da saúde foi associada à autoclassificação como branco/indígena/amarelo, ter consumo satisfatório de frutas e verduras e não ter feito consulta médica nos últimos 12 meses. Pior autoavaliação esteve associada aos indivíduos mais velhos, mais escolarizados, obesos, com maior número de doenças crônicas, com limitação nas atividades da vida diária, que informaram sentir depressão nos últimos 15 dias e entre aqueles com maior apoio social. Na análise estratificada por sexo, as variáveis consumo de frutas e apoio social perderam a significância. As demais apresentaram padrão semelhante à amostra total

Tabela 3

Associações de variáveis demográficas, socioeconômicas, comportamentais e de saúde com a autoavaliação da saúde de adultos: amostra total e estratificada por sexo. População quilombola, Vitória da Conquista, Bahia, Brasil, 2011.

\begin{tabular}{|c|c|c|c|c|c|c|}
\hline \multirow[t]{2}{*}{ Variáveis } & \multicolumn{2}{|c|}{ Total * } & \multicolumn{2}{|c|}{ Homens ** } & \multicolumn{2}{|c|}{ Mulheres ** } \\
\hline & OR & IC95\% & OR & IC95\% & OR & IC95\% \\
\hline \multicolumn{7}{|l|}{ Quilombola } \\
\hline Sim & 1,00 & & 1,00 & & 1,00 & \\
\hline Não & 1,02 & 0,$69 ; 1,49$ & 0,96 & 0,$57 ; 1,63$ & 1,14 & 0,$65 ; 1,98$ \\
\hline \multicolumn{7}{|l|}{ Idade (anos) } \\
\hline $18-29$ & 1,00 & & 1,00 & & 1,00 & \\
\hline $30-39$ & 0,81 & 0,$53 ; 1,22$ & 1,03 & 0,$53 ; 2,02$ & $0,68 * \star \star$ & 0,$40 ; 1,17$ \\
\hline $40-49$ & $0,55 \#$ & 0,$36 ; 0,84$ & $0,56 \# \#$ & 0,$29 ; 1,05$ & 0,55 \#\#\# & 0,$31 ; 0,97$ \\
\hline $50-59$ & $0,43 \S$ & 0,$27 ; 0,69$ & 0,47 \#\#\# & 0,$24 ; 0,92$ & 0,39 \# & 0,$20 ; 0,76$ \\
\hline 60 e mais & $0,32 \S$ & 0,$21 ; 0,49$ & $0,26 \S$ & 0,$14 ; 0,48$ & $0,42 \#$ & 0,$24 ; 0,75$ \\
\hline \multicolumn{7}{|l|}{ Sexo } \\
\hline Masculino & 1,00 & & & & & \\
\hline Feminino & 0,86 & 0,$65 ; 1,14$ & & & & \\
\hline \multicolumn{7}{|l|}{ Cor } \\
\hline Preta & 1,00 & & 1,00 & & 1,00 & \\
\hline Parda & 0,96 & 0,$71 ; 1,30$ & 0,98 & 0,$62 ; 1,53$ & 0,95 & 0,$63 ; 1,43$ \\
\hline Outra & $2,00 \#$ & 1,$31 ; 3,07$ & 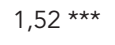 & 0,$84 ; 2,74$ & $2,80 \#$ & 1,$49 ; 5,26$ \\
\hline \multicolumn{7}{|l|}{ Escolaridade (anos) } \\
\hline 0 & 1,00 & & 1,00 & & 1,00 & \\
\hline $1-3$ & 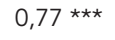 & 0,$52 ; 1,14$ & 1,03 & 0,$60 ; 1,75$ & 0,60 & 0,$33 ; 1,08$ \\
\hline $4-7$ & $0,74 * \star \star$ & 0,$49 ; 1,13$ & 1,13 & 0,$62 ; 2,05$ & 0,49 \#\#\# & 0,$27 ; 0,90$ \\
\hline 8 e mais & 0,70 & 0,$39 ; 1,24$ & 1,01 & 0,$45 ; 2,24$ & $0,47 \#$ & 0,$20 ; 1,08$ \\
\hline \multicolumn{7}{|l|}{ Situação conjugal } \\
\hline Sem companheiro(a) & 1,00 & & 1,00 & & 1,00 & \\
\hline Com companheiro(a) & 1,21 & 0,$90 ; 1,64$ & 1,21 & 0,$77 ; 1,90$ & 1,13 & 0,$75 ; 1,72$ \\
\hline \multicolumn{7}{|l|}{ Possuir fonte de renda } \\
\hline Não & 1,00 & & 1,00 & & 1,00 & \\
\hline Sim & 1,05 & 0,$76 ; 1,45$ & 1,14 & 0,$72 ; 1,79$ & 0,97 & 0,$61 ; 1,53$ \\
\hline \multicolumn{7}{|l|}{ Prática de atividade física } \\
\hline Não & 1,00 & & 1,00 & & 1,00 & \\
\hline Sim & 1,05 & 0,$75 ; 1,47$ & 1,08 & 0,$66 ; 1,77$ & 1,13 & 0,$70 ; 1,85$ \\
\hline
\end{tabular}

(continua) 
Tabela 3 (continuação)

\begin{tabular}{|c|c|c|c|c|c|c|}
\hline \multirow[t]{2}{*}{ Variáveis } & \multicolumn{2}{|c|}{ Total * } & \multicolumn{2}{|c|}{ Homens ** } & \multicolumn{2}{|c|}{ Mulheres ** } \\
\hline & OR & IC95\% & OR & IC95\% & OR & IC95\% \\
\hline Insatisfatório & 1,00 & & 1,00 & & 1,00 & \\
\hline Satisfatório & 1,22 *** & 0,$90 ; 1,65$ & 1,33 & 0,$85 ; 2,08$ & 1,18 & 0,$78 ; 1,78$ \\
\hline \multicolumn{7}{|l|}{ Estado nutricional } \\
\hline Sobrepeso & 0,96 & 0,$70 ; 1,33$ & 0,90 & 0,$56 ; 1,46$ & 1,01 & 0,$65 ; 1,57$ \\
\hline Obeso & 0,65 \#\# & 0,$40 ; 1,04$ & 0,24 \#\# & 0,$08 ; 0,72$ & 0,78 & 0,$45 ; 1,35$ \\
\hline \multicolumn{7}{|l|}{ Consumo de álcool } \\
\hline Nenhum & 1,00 & & 1,00 & & 1,00 & \\
\hline Moderado & 0,83 & 0,$60 ; 1,15$ & 0,96 & 0,$61 ; 1,52$ & $0,74 * \star \star$ & 0,$47 ; 1,16$ \\
\hline Fumante & 0,87 & 0,$58 ; 1,31$ & 1,03 & 0,$59 ; 1,78$ & 0,72 & 0,$36 ; 1,43$ \\
\hline \multicolumn{7}{|c|}{ Número doenças crônicas } \\
\hline \multicolumn{7}{|c|}{ (com diagnóstico médico) } \\
\hline Nenhuma & 1,00 & & 1,00 & & 1,00 & \\
\hline 1 & $0,34 \S$ & 0,$24 ; 0,48$ & $0,36 \S$ & 0,$22 ; 0,60$ & $0,31 \S$ & 0,$19 ; 0,50$ \\
\hline 2 & $0,17 \S$ & 0,$11 ; 0,27$ & $0,14 \S$ & 0,$07 ; 0,27$ & $0,19 \S$ & 0,$10 ; 0,36$ \\
\hline 3 ou mais & $0,10 \S$ & 0,$06 ; 0,17$ & $0,10 \S$ & 0,$04 ; 0,25$ & $0,09 \S$ & 0,$04 ; 0,19$ \\
\hline \multirow{2}{*}{\multicolumn{7}{|c|}{$\begin{array}{l}\text { Limitação nas atividades da } \\
\text { vida diária }\end{array}$}} \\
\hline & & & & & & \\
\hline Nenhuma & 1,00 & & 1,00 & & 1,00 & \\
\hline \multicolumn{7}{|l|}{ Acidentes/Ferimentos } \\
\hline \multicolumn{7}{|l|}{ (últimos 12 meses) } \\
\hline Não & 1,00 & & 1,00 & & 1,00 & \\
\hline $\operatorname{Sim}$ & 0,72 & 0,$46 ; 1,13$ & 0,73 & 0,$40 ; 1,35$ & 0,70 & 0,$36 ; 1,34$ \\
\hline \multicolumn{7}{|l|}{ Consulta médica (últimos } \\
\hline \multicolumn{7}{|l|}{12 meses) } \\
\hline Sim & 1,00 & & 1,00 & & 1,00 & \\
\hline Não & $2,02 \S$ & 1,$50 ; 2,71$ & $2,27 \S$ & 1,$44 ; 3,58$ & $1,78 \#$ & 1,$20 ; 2,64$ \\
\hline \multicolumn{7}{|l|}{ Apoio social } \\
\hline Menor apoio & 1,00 & & 1,00 & & 1,00 & \\
\hline Maior apoio & $0,78 \# \#$ & 0,$58 ; 1,06$ & 0,80 & 0,$52 ; 1,21$ & 0,82 & 0,$52 ; 1,28$ \\
\hline \multicolumn{7}{|l|}{ Contexto domiciliar } \\
\hline Piores condições & 1,00 & & 1,00 & & 1,00 & \\
\hline Melhores condições & 1,10 & 0,$83 ; 1,45$ & 0,98 & 0,$65 ; 1,47$ & 1,20 & 0,$82 ; 1,76$ \\
\hline
\end{tabular}

IC95\%: intervalo de 95\% de confiança; OR: odds ratio.

* Análise ajustada por sexo e idade;

** Análise ajustada por idade;

*** $p<0,20$;

$\# p<0,01$

$\#$ \# $<0,10$;

$\# \#$ \# $<0,05$;

$\S p<0,001$. 
com pequenas especificidades. Entre os homens a escolaridade perdeu a significância, e entre as mulheres o consumo moderado de álcool apresentou associação negativa com melhor autoavaliação da saúde.

A Tabela 4 apresenta o resultado dos ajustes dos modelos finais. Na interpretação dos modelos é necessário considerar dois grupos de variáveis explicativas: aquelas em que o pressuposto de chances proporcionais foi verificado e aque- las para as quais o pressuposto não foi verificado. Para o primeiro grupo o efeito da variável é constante em todas as transições e há somente um parâmetro a ser estimado. Para as demais, estima-se um coeficiente para cada transição.

Para a amostra total, atendendo ao pressuposto de chances proporcionais, verificouse uma melhor autovaliação da saúde entre as mulheres e entre os indivíduos que informaram consumo de frutas e verduras satisfatório. A as-

Modelos de odds proporcionais parciais (múltiplos) explicativos da autoavaliação da saúde de adultos: amostra total e estratificada por sexo. População quilombola, Vitória da Conquista, Bahia, Brasil, 2011.

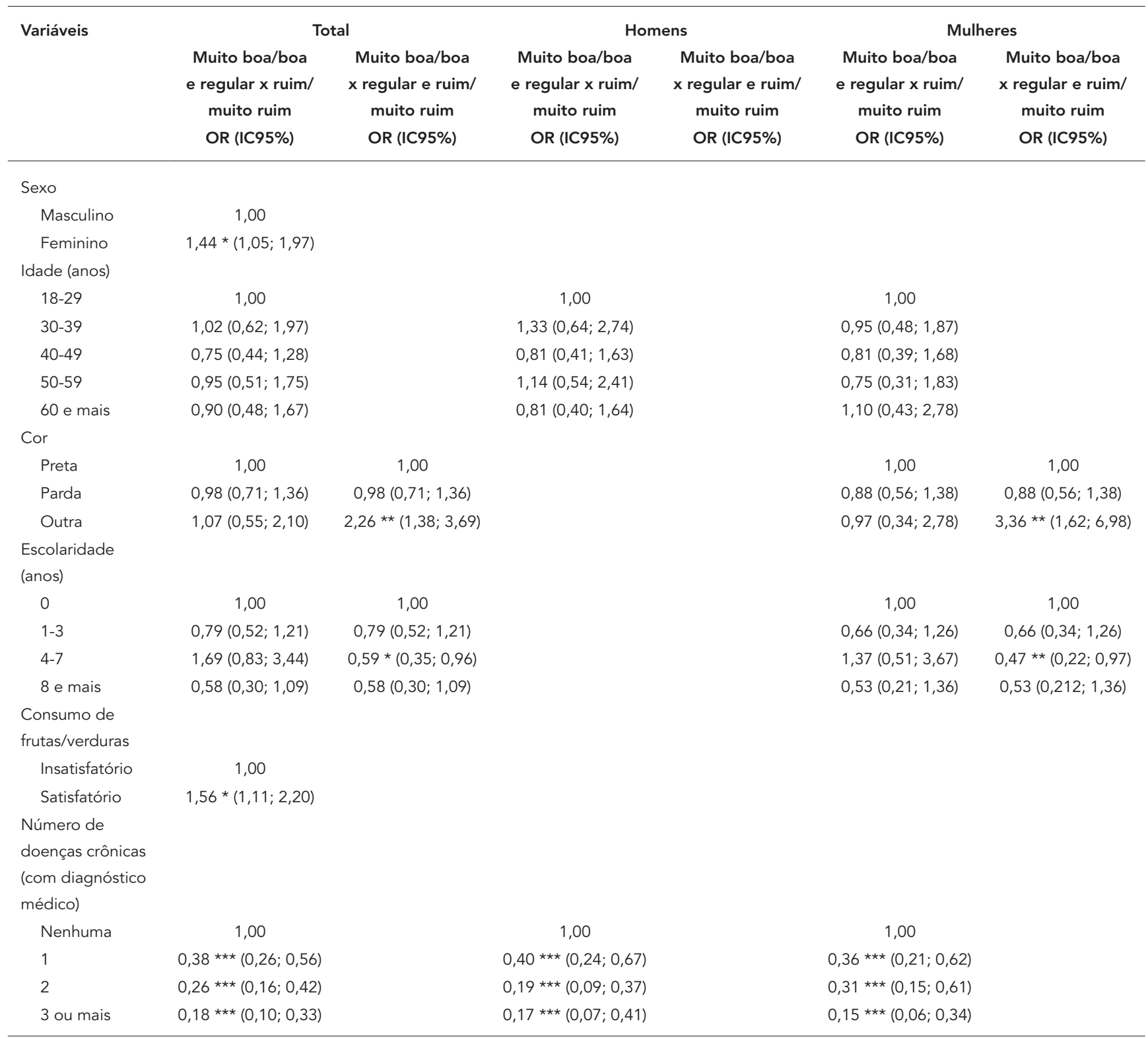

(continua) 
Tabela 4 (continuação)

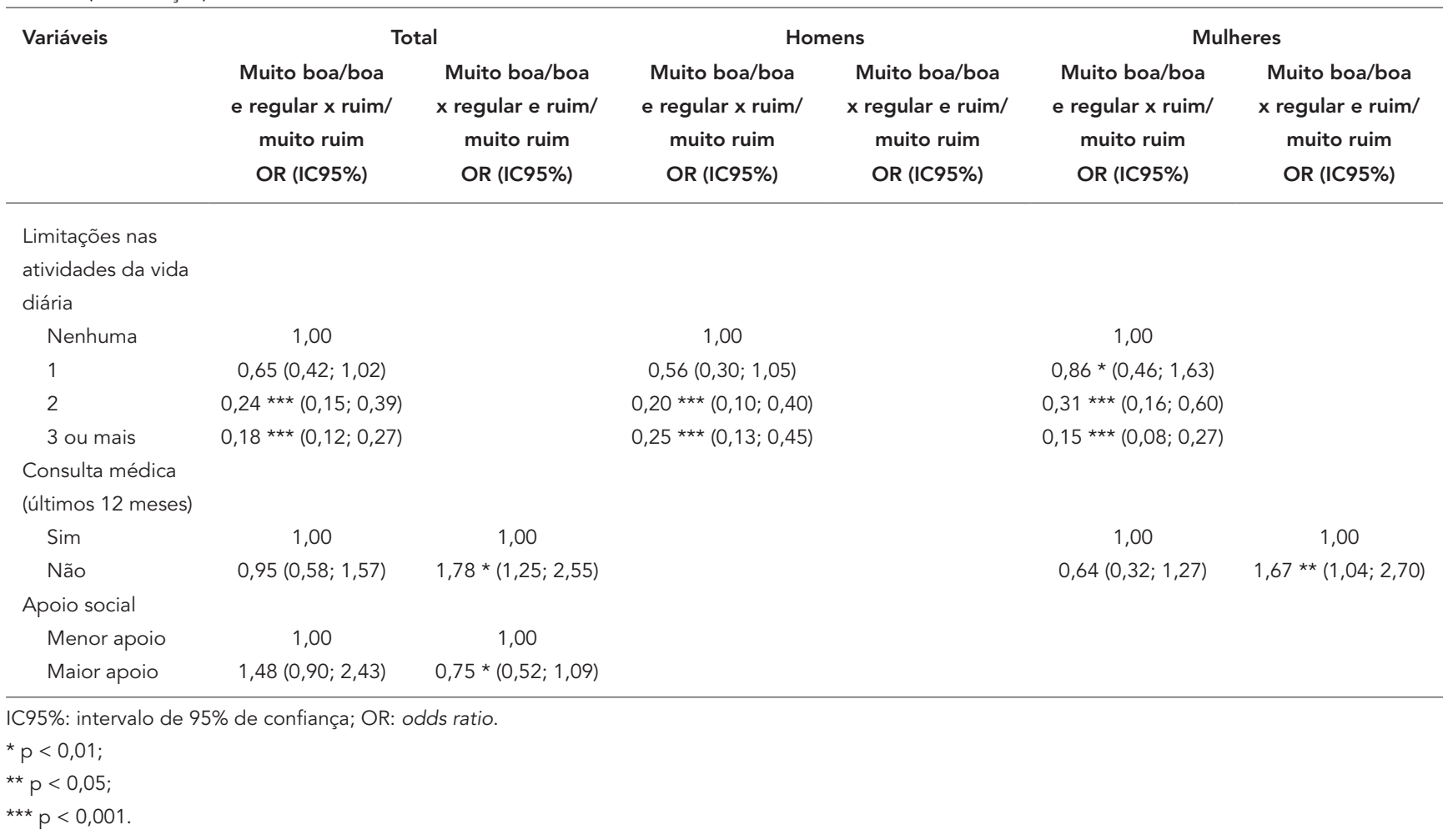

sociação da autoavaliação em saúde foi negativa com o número de doenças crônicas e a limitação das atividades da vida diária, a partir de duas limitações.

As variáveis cor, escolaridade, ter feito consulta médica e apoio social estiveram associadas apenas à chance de avaliar a saúde como muito boa/boa. Pessoas com 4 a 7 anos de estudos, aquelas que recebem maior apoio social apresentaram menor chance de reportar a saúde muito boa/boa, já as que se declararam brancas/ indígenas/amarelas e as que não consultaram o médico nos últimos 12 meses apresentaram maior chance de se avaliar nesta categoria.

A amostra de mulheres apresentou o mesmo comportamento da amostra total, exceto para a variável apoio social, que não se mostrou significativa em nenhum dos subgrupos analisados. Entre os homens, melhor autoavaliação em saúde esteve associada negativamente com o número de doenças crônicas e limitação das atividades diárias.

\section{Discussão}

Foi encontrada prevalência elevada de autoavaliação de saúde ruim/muito ruim $(12,5 \%)$ quando comparada a valores obtidos em outras pesquisas nacionais. Estudo utilizando os dados da Pesquisa Mundial de Saúde de 2003 reportou estimativa de $9 \%$ de autoavaliação ruim/muito ruim para a população adulta brasileira 7, e os resultados da PNAD $2008{ }^{9}$ informaram prevalência de 5,6\%, com variação entre os sexos - 5,9\% entre as mulheres e 5,3\% entre os homens, com pior autoavaliação de estado de saúde nos que residiam na área rural $(7,3 \%)$. Para a zona rural da Bahia, encontrou-se 4,8\% de autoavaliação ruim/muito ruim. Esses resultados são inferiores aos encontrados neste trabalho, cuja distribuição por sexo foi de $14 \%$ entre os homens e $11,1 \%$ entre as mulheres.

Comparando com a população brasileira em 2008 10, a população das comunidades quilombolas de Vitória da Conquista era mais jovem, apresentou perfil semelhante com relação ao tabagismo e à inatividade física, maior porcentagem de eutróficos, de consumo adequado de frutas e verduras e menos doenças crônicas. $\mathrm{O}$ nível de escolaridade era mais baixo, apresentou maior prevalência de consumo excessivo de álcool e a presença de negros e pardos era superior. Embora em muitos aspectos a população das comunidades quilombolas de Vitória da Conquista tenha apresentado perfil mais compatível com um estado de saúde mais favorável, a prevalência de autoavaliação negativa da saúde nesta popu- 
lação foi mais do que o dobro da observada na amostra da população brasileira ${ }^{9}$.

A autoavaliação de saúde é recomendada para uso em estudos populacionais por ser um indicador viável, inclusivo e informativo da situação de saúde, das necessidades e uso de serviços de saúde 5,6,8,9,10. A proporção de pessoas que define seu estado de saúde como regular ou ruim é poderoso no sentido de predizer o uso de serviços de saúde e estar associado à mortalidade em estudos longitudinais 1,3. Dessa maneira, a elevada prevalência de autoavaliação negativa de saúde informa a situação de precariedade social vivenciada por esta população quilombola.

Entre as variáveis sociodemográficas, a cor da pele esteve associada ao desfecho de interesse, na população total e entre as mulheres, com as pessoas classificadas como pretas ou pardas apresentando pior autoavaliação de saúde. Esses resultados corroboram achados que mostram uma pior autoavaliação de saúde entre minorias étnicas/raciais 18,20,26. Os negros apresentam piores resultados de saúde mesmo quando comparados a indivíduos brancos com os mesmos níveis socioeconômicos 20.

Encontrou-se melhor autoavaliação da saúde entre as mulheres, resultado distinto do encontrado em estudos nacionais 4,10. Pavão et al. 10 observam que a associação entre sexo e autoavaliação da saúde tem apresentado resultados conflitantes em estudos internacionais e nacionais 4,9,31. Macintyre et al. 31 alertam para o risco do excesso de generalização dos resultados encontrados para a associação entre sexo e saúde, ressaltando que complexidades nos padrões da relação estão sendo negligenciados.

Um resultado que se contrapõe a outros trabalhos foi a associação negativa identificada entre maior escolaridade e pior autoavaliação da saúde, observada para a amostra total e entre as mulheres. Consistentemente, estudos têm encontrado maior nível educacional associado a uma melhor saúde autorreferida 8,9,10. A associação entre escolaridade e saúde é explicada por meio de três vias inter-relacionadas: conhecimento sobre a saúde e adoção de comportamentos saudáveis; melhoria de emprego e renda; e maior controle de fatores sociais e psicológicos, levando à redução de estresse. Uma possível explicação para o resultado divergente encontrado no nosso estudo seria o baixo nível de escolaridade dessa população quilombola, em que observouse $32,3 \%$ dos indivíduos sem instrução formal e escolaridade mediana de três anos de estudos. Nesse ambiente, pode-se conjecturar que um pequeno aumento na escolaridade seja suficiente para modificar o contexto de avaliação, aumentando a expectativa com relação ao pró- prio estado de saúde, mas incapaz de traduzir em mudanças nas condições de vida e nas escolhas pessoais necessárias para atingir um melhor nível efetivo de saúde. Essa dissonância entre a expectativa e a ação poderia levar à relação negativa observada ${ }^{3}$.

Corroborando com os resultados de outros estudos, nacionais e internacionais, as variáveis relacionadas à situação de saúde - presença de doenças crônicas e atividades da vida diária comprometidas - estiveram negativamente associadas com a melhor autoavaliação de saúde $8,9,10$.

Quanto à consulta médica, observou-se uma relação positiva entre não consultar o médico e uma melhor autoavaliação de saúde, na população total e para as mulheres, indicando que para essa população a procura por consulta médica parece estar relacionada com a situação de doença. Em parte esse resultado é justificado pela dificuldade de acesso aos serviços de saúde que se observa nas comunidades rurais em geral e nesta, em particular ${ }^{32}$. Resultado semelhante foi encontrado por Kassouf 33, para o Brasil urbano e rural, em análise dos dados da PNAD 1998.

Entre os resultados obtidos neste estudo, alguns são especialmente reveladores. O primeiro deles diz respeito à composição dos modelos finais. Houve predomínio dos indicadores objetivos de saúde, sendo a dimensão física a única a permanecer no modelo masculino. Tomando o modelo de Jylhä ${ }^{3}$ como referencial teórico podese apreender que, de maneira geral, o conceito de saúde utilizado por essa população está fortemente relacionado à ausência de doenças e limitações nas atividades da vida diária. Uma possível explicação para esse comportamento seria a tendência observada em populações com menor escolaridade em considerar os aspectos físicos e funcionais para a autoavaliação de saúde, e grupos mais escolarizados tenderiam a apreciar aspectos multidimensionais na sua avaliação: sensação de bem-estar físico, psicológico, estar ativo fisicamente, entre outros 34 . Há diferença também por grupo etário, com pessoas mais jovens valorizando principalmente comportamentos relacionados à saúde e medidas objetivas de saúde, enquanto pessoas idosas tendem a considerar um contexto mais complexo ${ }^{3}$. A permanência das variáveis cor da pele, escolaridade e não realizar consultas médicas nos últimos 12 meses no modelo final feminino sugere que as mulheres, apesar de não diferirem dos homens com relação à escolaridade e apresentarem uma distribuição etária mais jovem, avaliam sua saúde utilizando um modelo mais abrangente. Essa diferença pode ser explicada pela articulação entre a percepção de saúde e 
gênero, compreendido como a construção social que considera, além das diferenças biológicas, as diferenças socioculturais e suas implicações como acesso diferenciado à informação, saúde, educação e ao poder de negociação nas relações sociais $15,35,36$.

Um segundo resultado singular é a permanência da variável cor da pele no modelo final do grupo das mulheres. As mulheres que se autoclassificaram como branca/indígena/amarela avaliaram melhor a sua saúde do que as mulheres que se classificaram como pretas ou pardas. Resultado semelhante foi obtido por Barata et al. 20 em estudo envolvendo a população brasileira de 15 a 64 anos de idade, com dados da PNAD 1998, em que foi reportado que mulheres negras possuem maior chance de referir saúde ruim, quando comparadas às mulheres brancas, mesmo após ajuste por idade, escolaridade e renda. Esses resultados informam a influência, entre outros fatores, das desigualdades raciais na autoavaliação do estado de saúde. Um terceiro resultado é a relação inversa apresentada entre apoio social e autoavaliação, que contraria outros estudos que apontam consistentemente para a associação entre melhor autoavaliação de saúde e maior apoio social 14. O resultado encontrado nos leva a inferir que um maior apoio social seja dispensado aos indivíduos mais frágeis, aqueles que estejam doentes ou têm alguma limitação funcional. Para melhor entender essa associação é preciso considerar que as variáveis de maior peso na composição do indicador apoio social foram os indicadores de presença de amigos e de familiares em que se confia. Para as variáveis relativas à participação em agremiações ou reuniões, grande parte da amostra respondeu nunca ou quase nunca participar e estas tiveram menor peso na formação do índice.

Finalmente, um ponto a se destacar refere-se à caracterização dos indivíduos com relação à autodeclaração de pertencimento ao grupo quilombola e sua associação com a autoavaliação em saúde. Considerando que $16,5 \%$ se autodeclararam não quilombolas e que estes não diferiram dos quilombolas com relação à autodeclaração do estado de saúde, há que se perguntar se os baixos níveis de saúde encontrados nesta população estão associados à situação da comunidade ser quilombola ou a outras características da comunidade: rural, pobre, do Nordeste brasileiro.

Dentre as limitações presentes neste estudo, pode-se citar a não inclusão de escalas de discriminação no instrumento de coleta de dados, o que impossibilitou o aprofundamento na discussão da associação da cor com os aspectos da saúde. Não foi possível confrontar nossos resultados com os achados relativos a outras populações re- manescentes de quilombos, pela inexistência de publicações sobre o tema. Mesmo a comparação com os resultados de outros grupos populacionais brasileiros fica de certa forma comprometida, uma vez que não há uniformidade nas alternativas de classificação da saúde apresentadas ao entrevistado, tampouco na agregação das categorias na etapa de análise. Outra limitação do estudo está relacionada com as interpretações da pergunta e da escala de respostas pré-definidas 22 . Considerando que a população quilombola inscreve-se em um contexto sociocultural específico, seria relevante investigar a adequação do instrumento de coleta de dados para este grupo populacional. Como em todo estudo transversal há a limitação relacionada ao delineamento, que torna inviável o estabelecimento de relações de temporalidade e assimetria.

Destaca-se o emprego do modelo para análise das associações. Levando-se em consideração a característica ordinal da variável dependente, não apenas a interpretação dos resultados se torna mais simples como o poder de discriminação do modelo fica aumentado. São raros os trabalhos que utilizam a regressão logística ordinal para analisar a autoavaliação do estado de saúde 9 .

Os resultados deste estudo permitem vislumbrar a situação de saúde precária dessas comunidades, revelada por uma elevada autopercepção de saúde muito ruim/ruim. Evidenciam, também, uma estrutura da autoavaliação de saúde na qual a dimensão física mostrou-se preponderante. Permitiram demonstrar a importância da inclusão da variável cor da pele nas pesquisas em saúde 23. Considerando que a autoavaliação da saúde é dependente não apenas das características do indivíduo, mas também do contexto social no qual ele está inserido, sugere-se a realização de estudos qualitativos que aprofundem o entendimento dos fatores associados à autoavaliação do estado de saúde e que permitam identificar a conotação atribuída às diferentes categorias de respostas pré-definidas, adequando-as ao contexto específico $3,9,22,34$.

O estudo mostrou também a necessidade de se comparar as comunidades quilombolas com outras comunidades rurais brasileiras, no intuito de procurar entender a real dimensão da situação dos remanescentes de quilombos.

Ressalta-se, também, a relevância de mais pesquisas envolvendo comunidades remanescentes de quilombos no país, com delineamentos que possibilitem o reconhecimento de suas especificidades, e colaborem na construção e implementação de políticas públicas que contribuam no enfrentamento das desigualdades raciais e redução da vulnerabilidade social destas comunidades. 


\section{Resumen}

Este estudio se centra en analizar la prevalencia de la autoevaluación de salud negativa y factores asociados, en la población quilombola de Vitória de la Conquista, Bahía, Brasil. Se realizó una encuesta domiciliaria con 797 adultos, en 2011. Los datos sobre la autoevaluación de salud, condiciones socioeconómicas y demográficas, estilo de vida, apoyo social, situación de salud y acceso a servicios de salud, fueron obtenidos a través de la cumplimentación de cuestionarios. El análisis multivariable se realizó por regresión logística ordinal, ajustada por sexo y edad. La prevalencia de la autoevaluación negativa de salud fue de un 12,5\%. Tras el modelo estadístico, las siguientes variables permanecieron asociadas a la evaluación de salud: color de piel, escolaridad, consumo satisfactorio de frutas y verduras, presencia de enfermedades crónicas, limitaciones físicas, apoyo social y al menos una consulta médica en los últimos 12 meses. La autoevaluación de salud se asoció a las dimensiones: socioeconómicaldemográfica, hábitos de vida, apoyo social y situación de salud.

Grupo de Ascendencia Continental Africana; Autoevaluación; Estado de Salud

\section{Colaboradores}

C. N. Kochergin participou da elaboração do projeto, planejamento e supervisão da coleta dos dados, revisão da literatura, análise dos dados e redação do artigo. F. A. Proietti colaborou na análise dos resultados e revisão final do artigo. C. C. César contribuiu na elaboração e orientação do projeto, revisão da literatura, análise dos dados e redação do artigo.

\section{Agradecimentos}

Aos pesquisadores do Projeto COMQUISTA pela participação na elaboração do projeto, planejamento e supervisão da coleta dos dados. À Secretaria Municipal de Saúde de Vitória da Conquista, especialmente aos profissionais de saúde responsáveis pelas comunidades quilombolas pesquisadas, pelo apoio oferecido para a realização do trabalho; e aos quilombolas entrevistados, pela receptividade. À Capes (Edital 05/2009); Ministério da Saúde/CNPq/FAPESB/SESAB (Edital 20/2010/PPSUS-BA), pelo financiamento. Ao CNPq pelas bolsas de produtividade concedidas à F. A. Proietti e C. C. César.

\section{Referências}

1. Idler EL, Benyamini Y. Self-rated health and mortality: a review of twenty-seven community studies. J Health Soc Behav 1997; 38:21-37.

2. Theme-Filha MM, Szwarcwald CL, Souza-Júnior PRB. Socio-demographc characteristics, treatments coverage and self-rated health of individuals who reported six chronic diseases in Brazil, 2003. Cad Saúde Pública 2005; 21 Suppl 1:S43-53.

3. Jylhä M. What is self-rated health and why does it predict mortality? Towards a unified conceptual model. Soc Sci Med 2009; 69:307-16.
4. Viacava F. Acesso e uso de serviços de saúde pelos brasileiros. Radis: Comunicação em Saúde 2010; 96:12-9.

5. Dachs JNW, Santos APRS. Autoavaliação do estado de saúde no Brasil: análise dos dados da PNAD/2003. Ciênc Saúde Coletiva 2006; 11:887-94.

6. Lima-Costa MF, César CC, Chor D, Proietti FA. Selfrated health compared with objectively measured health status as a tool for mortality risk screening in older adults: 10-year follow-up of the Bambuí Cohort Study of Aging. Am J Epidemiol 2012; 175:228-35. 
7. Szwarcwald CL, Souza-Júnior PR, Esteves MA, Damacena GN, Viacava F. Socio-demographic determinants of self-rated health in Brasil. Cad Saúde Pública 2005; 21 Suppl 1:S54-64.

8. Barros MBA, Zanchetta LM, Moura EC, Malta DC. Auto-avaliação de saúde e fatores associados, Brasil 2006. Rev Saúde Pública 2009; 43 Suppl 2:S27-37.

9. Moraes JR, Moreira JPL, Luiz RR. Associação entre o estado de saúde autorreferido de adultos e a área de localização do domicílio: uma análise de regressão logística ordinal usando a PNAD 2008. Ciênc Saúde Coletiva 2011; 16:3769-80.

10. Pavão AL, Werneck GL, Campos MR. Autoavaliação do estado de saúde e a associação com fatores sociodemográficos, hábitos de vida e morbidade na população: um inquérito nacional. Cad Saúde Pública 2013; 29:723-34.

11. Franks, P, Gold MR, Fiscella K. Sociodemographics, self-rated health, and mortality in the US. Soc Sci Med 2003; 56:2505-14.

12. Kómár M, Nagymajtényi L, Nyári T, Paulik E. The determinants of self-rated health among ethnic minorities in Hungary. Ethn Health 2006; 11:121-32.

13. Lim WY, Stefan MA, Derrick H, Vineta B, Suok K. Gender, ethnicity, health behaviour \& self-rated health in Singapore. BMC Public Health 2007; $7: 184$.

14. Lindén-Boström M, Persson C, Eriksson C. Neighbourhood characteristics, social capital and selfrated health - a population-based survey in Sweden. BMC Public Health 2010; 10:628.

15. Denton M, Prus S, Walters V. Gender differences in health: a Canadian study of the psychosocial. Soc Sci Med 2004; 58:2585-600.

16. Henriques R. Desigualdade racial no Brasil: evolução das condições de vida na década de 90. Brasília: Instituto de Pesquisa Econômica Aplicada; 2001. (Texto para Discussão, 807).

17. Oliveira F. Saúde da população negra: Brasil ano 2001. Brasília: Organização Pan-Americana da Saúde; 2003.

18. Guerrero AFH, Silva DO, Toledo LM, Guerrero H, Teixeira P. Mortalidade infantil em remanescentes de quilombos do Município de Santarém - Pará, Brasil. Saúde Soc 2007; 16:103-10.

19. Volochko A, Batista LE. Saúde nos quilombos. São Paulo: Instituto de Saúde, Secretaria de Estado de Saúde de São Paulo; 2009. (Temas em Saúde Coletiva, 9).

20. Barata RB, Almeida MF, Montero CV, Silva ZP. Health inequalities based on ethnicity in individuals aged 15 to 64, Brazil, 1998. Cad Saúde Pública 2007; 23:305-13.

21. Travassos C, Williams DR. The concept and measurement of race and their relationship to public health: a review focused on Brazil and the United States. Cad Saúde Pública 2004; 20:660-78.

22. Chiavegatto Filho ADP, Laurenti R. Disparidades étnico-raciais em saúde autoavaliada: análise multinível de 2.697 indivíduos residentes em 145 municípios brasileiros. Cad Saúde Pública 2013; 29:1572-82.
23. Chor D, Lima CRA. Aspectos epidemiológicos das desigualdades raciais em saúde no Brasil. Cad Saúde Pública 2005; 21:1586-94.

24. Coimbra Jr. CEA, Santos RV. Saúde, minorias e desigualdade: algumas teias de inter-relações, com ênfase nos povos indígenas no Brasil. Ciênc Saúde Coletiva 2000; 5:125-32.

25. Amorim IG, Germani GI. Quilombos da Bahia: presença incontestável. In: Anais do X Encontro de Geógrafos da América Latina. São Paulo: Universidade de São Paulo; 2005. p. 796-812.

26. Presidência da República. Decreto no 4.887, de 20 de novembro de 2003. Regulamenta o procedimento para identificação, reconhecimento, delimitação, demarcação e titulação das terras ocupadas por remanescentes das comunidades quilombolas de que trata o art. 68 do Ato das Disposições Constitucionais Transitórias. Diário Oficial da União 2003; 21 nov.

27. Secretaria Especial de Políticas de Promoção da Igualdade Racial. Relatório de avaliação do plano plurianual 2008-2011: ano base 2008. Brasília: Secretaria Especial de Políticas de Promoção da Igualdade Racial; 2009.

28. Jardim R, Barreto SM, Giatti L. Auto-relato de informante secundário na avaliação da saúde de idosos. Rev Saúde Pública 2010; 44:1120-9.

29. Bezerra VM, Medeiros DS, Gomes KO, Souzas R, Giatti L, Steffens AP, et al. Inquérito de Saúde em Comunidades Quilombolas de Vitória da Conquista/BA (Projeto COMQUISTA): aspectos metodológicos e análise descritiva. Ciênc Saúde Coletiva 2014; 19:1835-47.

30. Abreu MNS, Siqueira AL, Caiaffa WT. Regressão logística ordinal em estudos epidemiológicos. Rev Saúde Pública 2009; 43:183-94.

31. Macintyre S, Hunt K, Sweeting H. Gender differences in health: are things really as simple as they seem? Soc Sci Med 1996; 42:617-24.

32. Gomes KO, Reis EA, Guimarães MDC, Cherchiglia ML. Utilização de serviços de saúde por população quilombola do Sudoeste da Bahia, Brasil. Cad Saúde Pública 2013; 29:1829-42.

33. Kassouf AL. Acesso aos serviços de saúde nas áreas urbana e rural do Brasil. Revista Economia e Sociologia Rural 2005; 43:29-44.

34. Huisman M, van Lenthe F, Mackenbach I. The predictive ability of self-rated health for mortality in different educational groups. Int J Epidemiology 2007; 36:1207-13.

35. Pinheiro RS, Viacava F, Travassos C, Brito AS. Gênero, morbidade, acesso e utilização de serviços de saúde no Brasil. Ciênc Saúde Coletiva 2002; 7:687-707.

36. Vintém JM. Inquéritos Nacionais de Saúde: autopercepção do estado de saúde: uma análise em torno da questão de gênero e da escolaridade. Rev Port Saúde Pública 2008; 26:5-16.

Recebido em 01/Ago/2013

Versão final reapresentada em 09/Dez/2013

Aprovado em 14/Jan/2014 